NASA Technıcal Memorandum 85989

NASA-TM-85989 19840020676

\title{
A Practical Adaptive-Grid Method for Complex Fluid-Flow Problems
}

\section{Kazuhiro Nakahashi and George S. Deiwert}

\section{LIBRARY ROPY}

$$
\text { j: ?: } 1984
$$

LANGLEY RESEART $W$ CENTER LIERARY, NASA HAMPTON. VIRGINIA

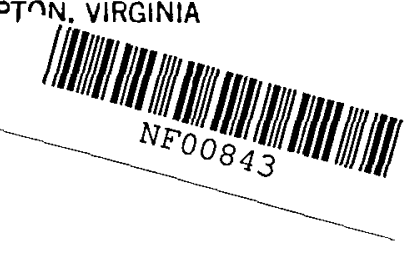


NASA Technıcal Memorandum 85989

\section{A Practical Adaptive-Grid Method for Complex Fluid-Flow Problems}

Kazuhıro Nakahashi,

George S. Deıwert, Ames Research Center, Moffett Field, Calıfornıa

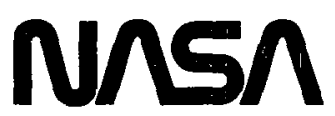

National Aeronautics and

Space Admınıstration

Ames Research Center

Moffett Field, Calıfornia 94035

$N 84-28745^{\neq}$ 


\title{
A Practical Adaptive-Grid Method for Complox Fluid-Flow Problems
}

\author{
Kazuhiro Nakahashi aNd George S. DeIwert \\ NASA Ames Research Center \\ Moffett Field, California 94035, U.S.A.
}

\section{INTRODUCTION}

Adaptive-grid methods are an important subject of study in computational finid dynamics becanse of their potential for improving the efficiency and acenracy of numerical methods. Various papers on grid adaptation have been presented in recent years, but few methods have been applied in practical applications.

In this paper, a practical solution-adaptive-grid method utilizing a tension and torsion spring analogy is proposed for multidimensional tluid-flow problems. The tension spring, which connects adjacent grid points to each other, controls grid spacings so that clustering is realized in regions containing shock waves and shear layers. The torsion spring, which is attached to each grid node, controls inclinations of coordinate lines and prevents excessive grid skewness. A marching procedure is used that results in a simple tridiagonal system of equations at each coordinate line to determine grid-point distribution. Multidirectional grid adaptation to the flow solution is achieved by successive applications of one-directional adaptation. Examples of applications for axisymmetric afterbody flow fields and two-dimensional transonic airfoil flow fields are shown.

\section{Grid adaptation in One Coordinate DiRection}

For simplicity of illnstration, consider grid adaptation to a flow field in which grid points are free to move along each $\eta$-coordinate line whose configuration is fixed. Let a grid point $A$ in figure 1 be connected to its adjacent points, $B$ and $C$, by tension springs whose spring constants are $K_{1,-1}$ and $K_{i, j}$. To distribute grid points along the $\eta_{i}$-coordinate line in proportion to the gradient of selected flow properties, the relationship between the spring constant $K$ and the gradient of the dependent variable $f$

$$
K_{i, j}=1+C_{1}\left|f_{i, j+1}-f_{i, j}\right| /\left(s_{i, j+1}-s_{i, j}\right)
$$

is used, where $C_{1}$ is a constant and $\theta_{3, j}$ is the are length calculated from point $(i, 1)$ along the $\eta_{1}$-coordinate.

Using equation (1), the distribution of grid points along the $\eta_{1}$-coordinate line, namely, new values of $s_{i, j}$, is determined by

$$
K_{0, j}\left(\theta_{1, j+1}-\theta_{1, j}\right)-K_{1, j-1}\left(\theta_{1, j}-\theta_{1, j-1}\right)=0
$$

The idea of a spring analogy represented in equation (1) was introduced by Gnoffo(ref. 1). In his model, the distribation of points along the $\eta_{1}$-coordinate is determined only by the gradients of llow properties along that coordinate line and is not affected by the distribution on adjacent $\eta$-coordinates, $\eta_{1-1}$ and $\eta_{3+1}$. This can lead to excessive skewness of grid lines, especially when applied to complex flow fields, and this lack of control of grid inclination makes it difficult to extend the scheme to more than one family of coordinates.

A force to control inclinations of $\xi$-coordinates in addition to that of grid spacings on $\eta$-coordinates will correct this deficiency. This control force can be given by considering torsion springs attached to nodes along the $\eta_{1-1}$ line. The torsion spring enforces the inclination of line $\overline{D A}$ to that of a reference line. If the spring constant of the torsion spring is denoted by $B$, a mathematical statement of the force is

$$
F_{\text {tormion }}=-B_{i-1,1}\left(\theta_{D A}-\phi\right)
$$

where $\theta_{D A}$ is a inclination of line $\overline{D A}$ and $\phi$ the inclination of the reference line. The reference line can be chosen as an extension of $\overline{F D}$ to avoid kinks in the $\xi$-line at point $D$, as a line normal to the $\eta_{i}$-coordinate to make the grid quasi-orthogonal, or as a streamline, and so forth. In practical calculations, a combination of these reference lines is used. The torsion spring constant $H$ can be prescribed for each coordinate line.

A balance equation for the complete spring system is

$$
K_{i, j}\left(s_{i, j+1}-8_{i, j}\right)-K_{i, j-1}\left(8_{1, j}-s_{i, j-1}\right)-H_{1-1, j}\left(\theta_{1-1, j}-\phi_{i-1, j}\right)=0
$$


To facilitate solutions to equation (4), the thind term is rewritten

$$
H_{i-1, j}\left(\theta_{z-1, j}-\phi_{i-1, j}\right) \Rightarrow \bar{H}_{i-1, j}\left(\theta_{i, j}-\bar{\theta}_{i, j}\right)
$$

where $\bar{\theta}_{1, y}$ is arc length to the intersection of reference line $\overline{D A^{\prime}}$ with the $\eta_{1}$-coordinate as depicted in figure 2. The $\bar{B}_{1}-1$, term is set equal to $B_{3}-1$, divided by length of $\overline{D A^{\prime}}$. Finally, equation (4) redaces to the following equation:

$$
K_{i, j-1 \theta_{1, j-1}}-\left(K_{i, j}+K_{i, j-1}+\bar{H}_{2-1, j}\right) \theta_{i, j}+K_{i, j} \theta_{i, j+1}=-\bar{H}_{3-1, j} \bar{\theta}_{i, j}
$$

This is a tridiagonal system of equations for $\theta_{2,}$ and can be readily solved.

In this analysis only the torsion force on the npstream side $\left(\eta_{3}-1\right)$ influences the distribution at $\eta_{2}$. This permits simple marching schemes to be used, without any loss of generality, and contributes to the simplicity and robustness of the method. Conversely, if the influence from both sides $\left(\eta_{1-1}\right.$ and $\left.\eta_{1+1}\right)$ is considered simultaneously, the computational effort is increased considerably withont any additional benefit. Note, too, that the downstream influence, $\left(\eta_{s+1}\right)$, conld be used instead of the upstream, without any additional complexity or loss of generality.

\section{Grid Adaptation IN MUlticoordinate DiRECTion}

A model asing tension and torsion springs for a two-directional adaptation can be depicted as in figare 3(a). Since each grid point is connected to its four adjacent points, the procedure for the grid movements of this model is more complicated and requires more computational effort. To minimize this complexity, a split model, which is a combination of one-directional adaptation, is used(fig. 3(b)). Grid movement is achieved by successive applications of the one-directional adaptation method. This is analogous to ADI schemes for partial differential equations. It is not necessary to achieve convergence in this adaptation procedure and, in fact, one iteration is sufficient in practical applications of the scheme.

The solntion field is interpolated onto the newly adapted grid, using second-order, one-dimensional Lagrange interpolation after each one-directional adaptation. Before this interpolation, it is possible to add or delete grid points at the users discretion, thus enhancing the method without any loss of accuracy or increased complexity. The successive application of one-directional adaptation also enhances the applicability of the method, in that unidirectional adaptation can be used in regions where gradients are large in onty one direction. Extension of this scheme to three-dimensions is straightforward.

\section{RESULTS}

Shown in figures 4-8 are examples of applications of the method to axisymmetric, plnme flow fields. These complex flow fields exhibit oblique shocks, barrel shocks, and slip surfaces, where grid points should be clustered for adequate resolution. The locations of these discontinuities are not known apriori, and a solntion-adaptive grid is particularty useful in realizing an efficient and accurate simulation.

The initial grid(fig. 4) was generated by an algebraic method and the flow field determined using a code(ref. 2) for the thin-layer Navier-Stokes equations. The free-stream Mach number is 2.01, the jet-exit Mach number is 2.5, and the static pressure ratio of the exhaust jet to the free-stream is 1 . The density gradient was chosen as a reference variable for the grid clustering, and figure 5 shows a solution-adapted grid which clearly has clustered points to the oblique shock, the slip surface, and the barrel shock. Shown in figure 6 are computed density contours using the grid shown in figure 5.

Shown in figure 7 is an adapted grid for a high-jet-pressure case (pressure ratio of 6), and density contours are shown in figure 8. The same initial grid(fig. 4) used in the previons case was used here.

The grids were adapted to the flow-field solutions periodically (typically two or three times) during the course of reaching a steady-state solution. In this way, the time required for adaptation is a negligible fraction of the total time required for flow-field solution.

Examples of the application to transonic thow fields past a NACA0012 airfoil are shown in figures 9-12. The free-stream Mach number is 0.8 and the angle of attack is $1.25^{\circ}$. Two-dimensional Enlef equations were solved, using the code ARC2D(refs. 3,4). Figare 9 shows an initial 0 -grid generated by an algebraic method. Grid-point distributions along $\xi$-coordinate lines, which are parallel to the airfoil surface, were adapted to the density gradients in the flow-field solution. Shown in figure 10 is an adapted grid showing appropriate clustering of grid points to the shocks. Computed Mach contours obtained using the adapted grid are shown in figure 11. Figure 12 shows a comparison of pressure coefficients obtained using nonadapted and adapted grids. This figure shows that shock waves are crisply resolved using the adapted grid.

An example for supersonic tow is shown in figures 13-15. The free-stream Mach number is 1.2 and the angle of attack is $1.25^{\circ}$. In this case, both $\xi$ and $\eta$-coordinate lines were adapted to the density gradients. 


\section{SUMMARY}

The principal features of the proposed adaptive-grid method are as follows:

1. It is a simple concept consisting of a tension and torsion spring analogy. The combination of these springs produces a suitable adaptive grid without excessive grid skewness.

2. A marching-type of calculation procedure minimizes the computation time.

3. The split-solution procedure for multidirectional adaptation is simple and practical.

4. The method can be applied independently to selected parts of the entire grid.

5. The controllability of grid inclinations with the torsion spring makes it possible to generate a nearly orthogonal adaptive grid. Also, the inclinations of grid lines near boundary can be specified arbitrarily.

These features make the proposed adaptive-grid method practical and robust, and enhance its applicability. The method can be applied to two- and three-dimensional finid-flow problems without any difficulty and requires little computational time and effort.

\section{REFERENCES}

1. Gnoffo, P. A.: A Finite-Volume, Adaptive Grid Algorithm Applied to Planetary Entry Flowfields, AIAA J., vol.21, no.9, 1983 , pp. 1249-1254.

2. Deiwert, G. S.; Andrews, A. E.; and Nakahashi, K.: Theoretical Analysis of Aircraft Afterbody Flow, AIAA Paper 84-1524, 1984.

3. Pulliam, T. H.; Jespersen, D. C.; and Childs, R. E.: An Enhanced Version of an Implicit Code for the Euler Equations, AIAA Paper 83-0344, 1983.

4. Polliam, T. H.: Euler and Thin Layer Navier-Stokes Codes : ARC2D, ARC3D, Notes for Computational Fluid Dynamies User's Workshop, The University of Tennessee Space Institute, Tullahoma, Tenn., Mar. 1984.

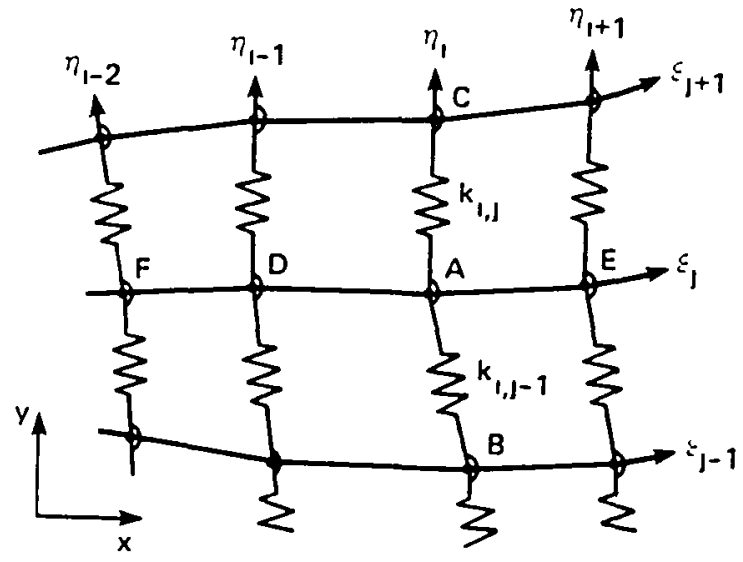

Fig.1 Schematic of adaptive-grid algorithm with tension and torsion spring analogy.

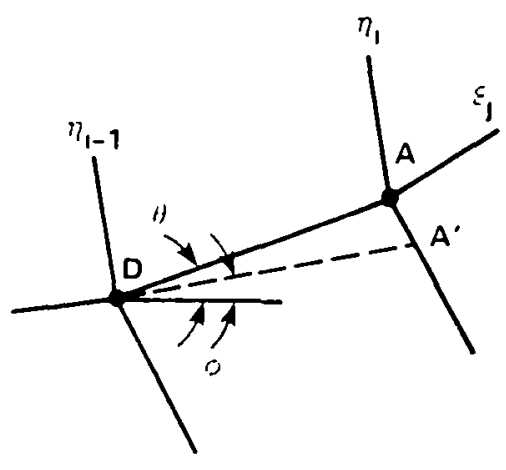

Fig.2 Notations for torsion spring analogy.

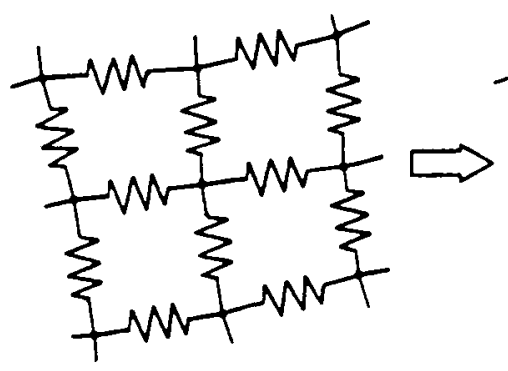

(a) Two-dimensional model.
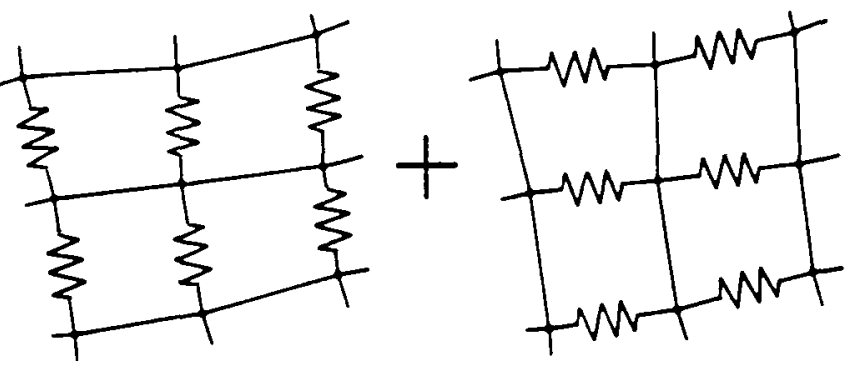

(b) Split model.

Fig.3 Moltidirectional adaptation. 


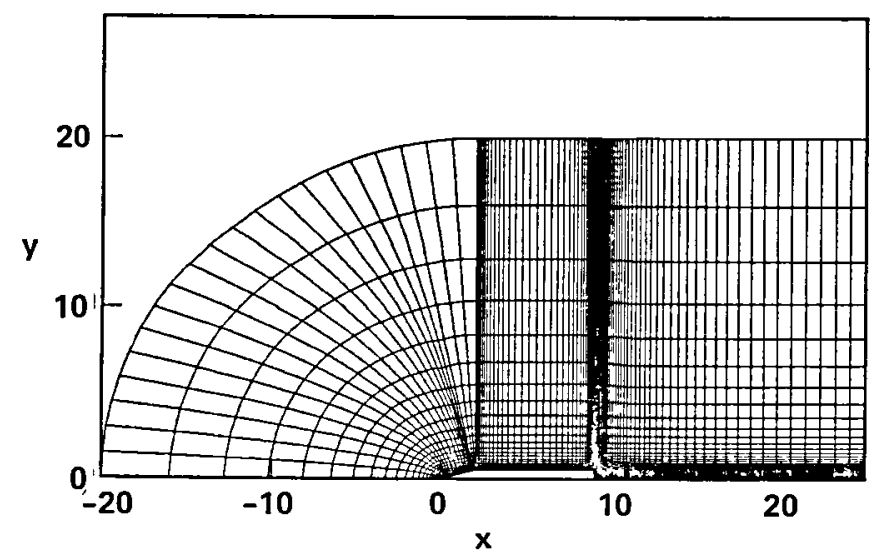

(a) Complete configuration.

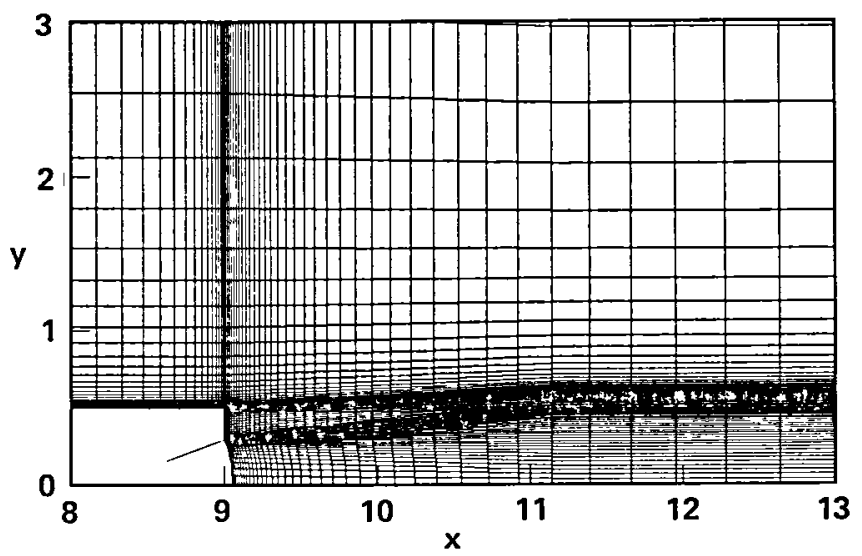

(b) Base region detail.

Fig.4 Initial grid for afterbody flow field.

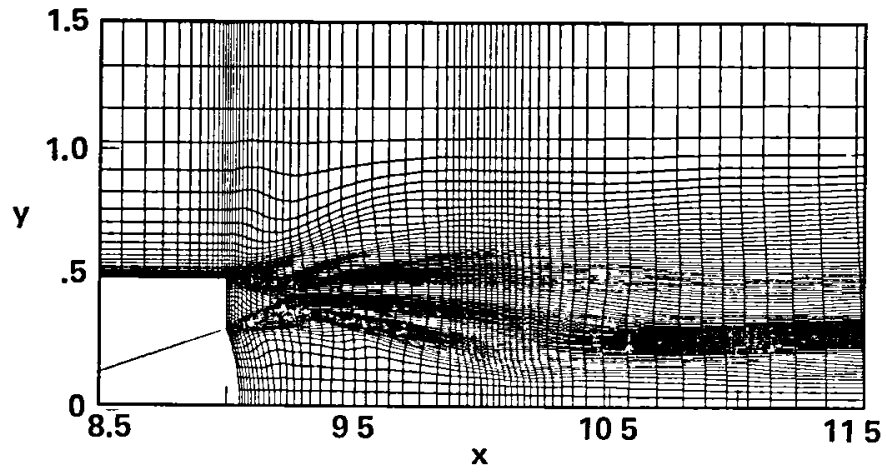

Fig.5 Adapted grid for afterbody flow field: $M_{\infty}=2.01, M_{J}=2.5: P_{J} / P_{\infty}=1.0$.

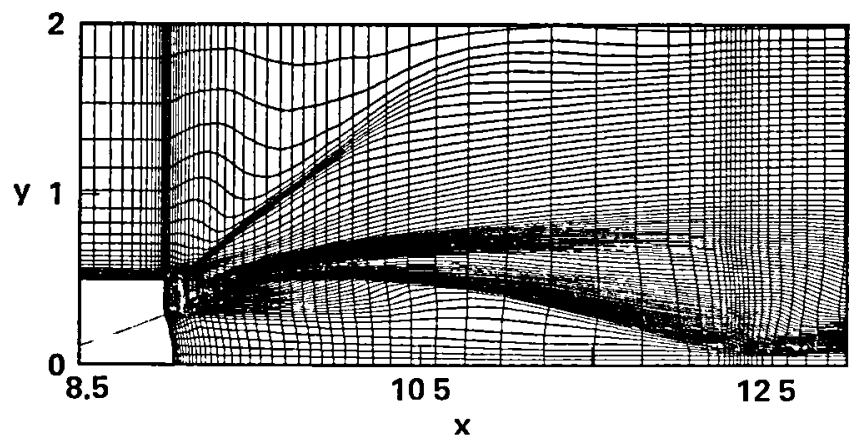

Fig.7 Adapted grid for afterbody flow field: $M_{\infty}=2.01, M_{J}=25: P_{J} / P_{\infty}=6.0$.

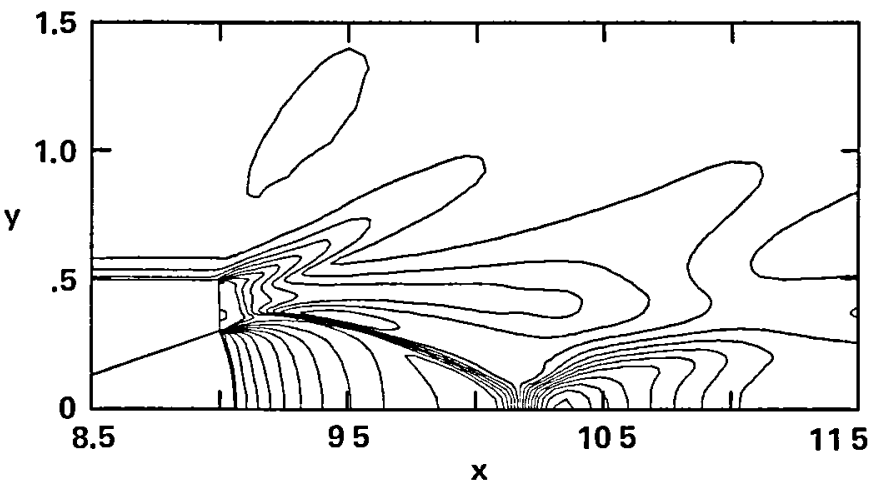

Fig.6 Compated density contours with adapted grid: $M_{\infty}=2.01, M_{J}=2.5 . P_{J} / P_{\infty}=1.0$.

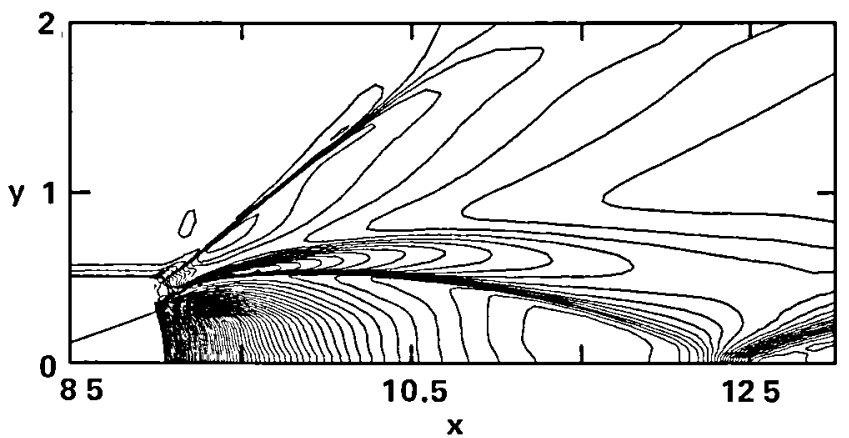

Fig.8 Computed density contonrs with adapted ond: $M_{\infty}=2.01, M_{J}=2.5: P_{J} / P_{\infty}=6.0$. 




Fig.9 Initial 0.grid for NACA0012 airfoil.

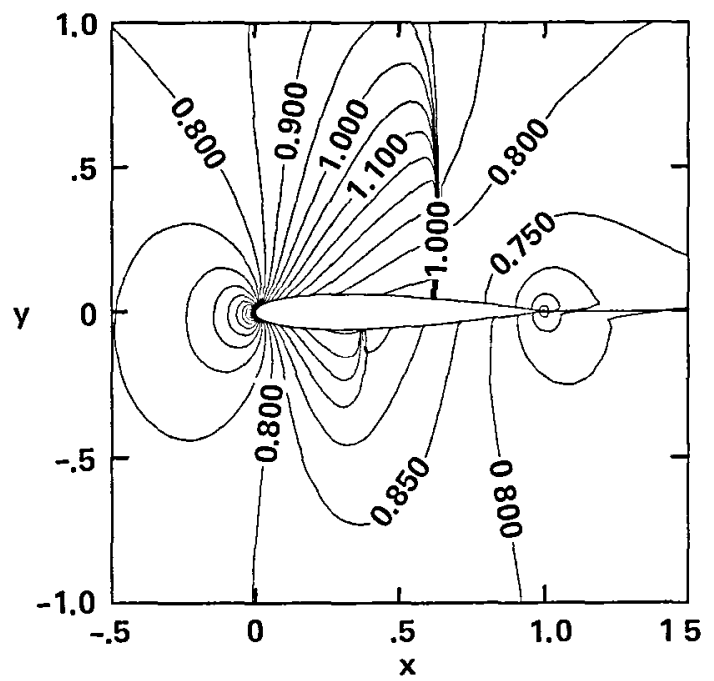

Fig.11 Computed Mach contours with adapted grid: $M_{\infty}=0.8, \alpha=1.25^{\circ}$.

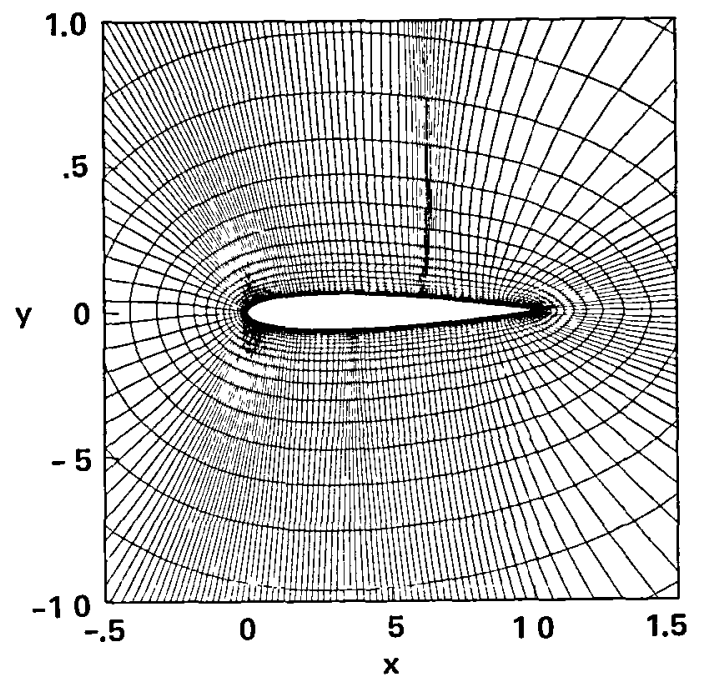

Fig.10 Adapted grid: $M_{\infty}=0.8, \alpha=1.25^{\circ}$.

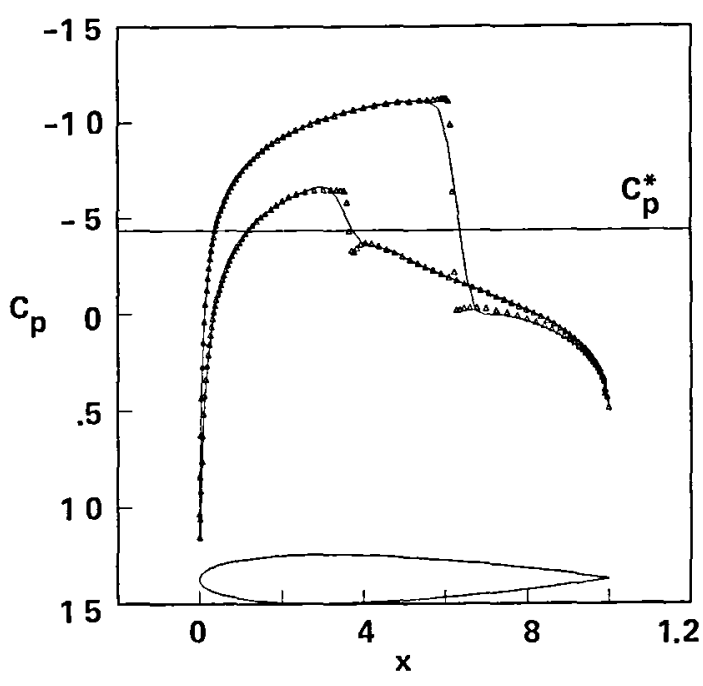

Fig.12 Cp for solutions with(dot) and without(line) adapted grid.

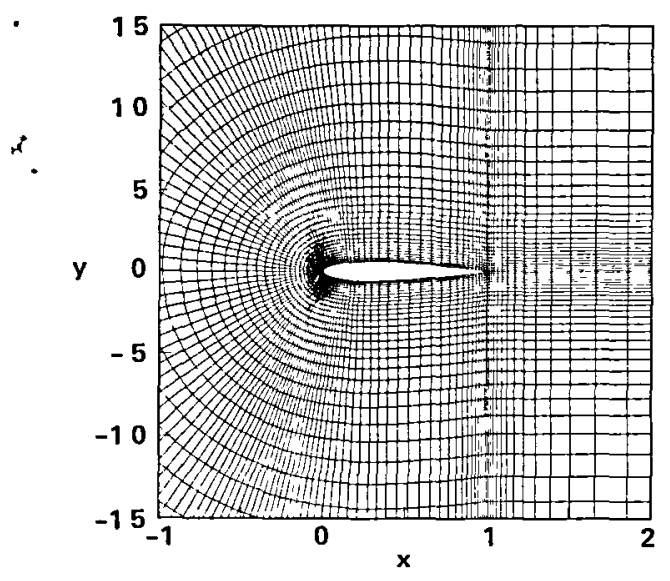

Fig.18 Initial C-grid.

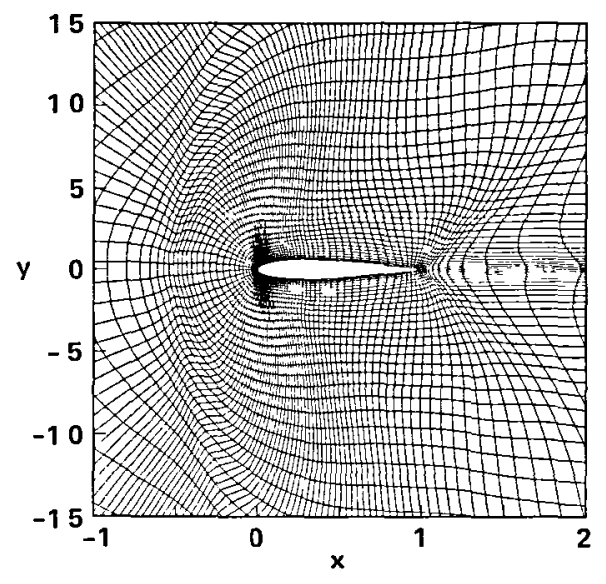

Fig.14 Adapted grid: $M_{\infty}=1.2, \alpha=1.25^{\circ}$.

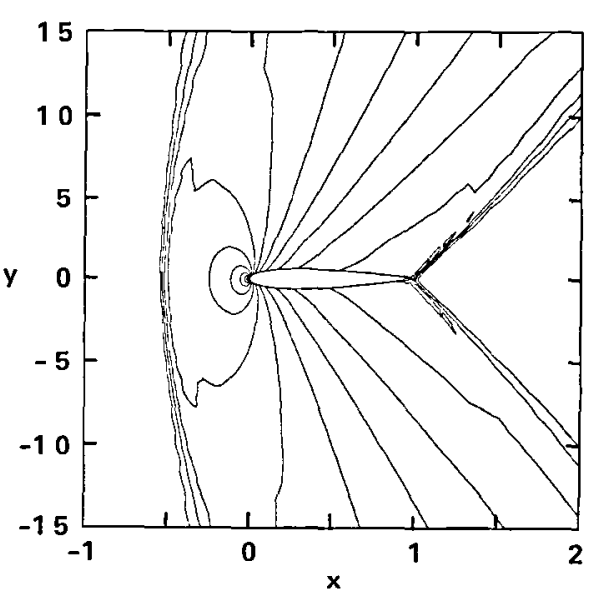

Fig.15 Computed Mach contours: $M_{\infty}=1.2, \alpha=1.25^{\circ}$. 


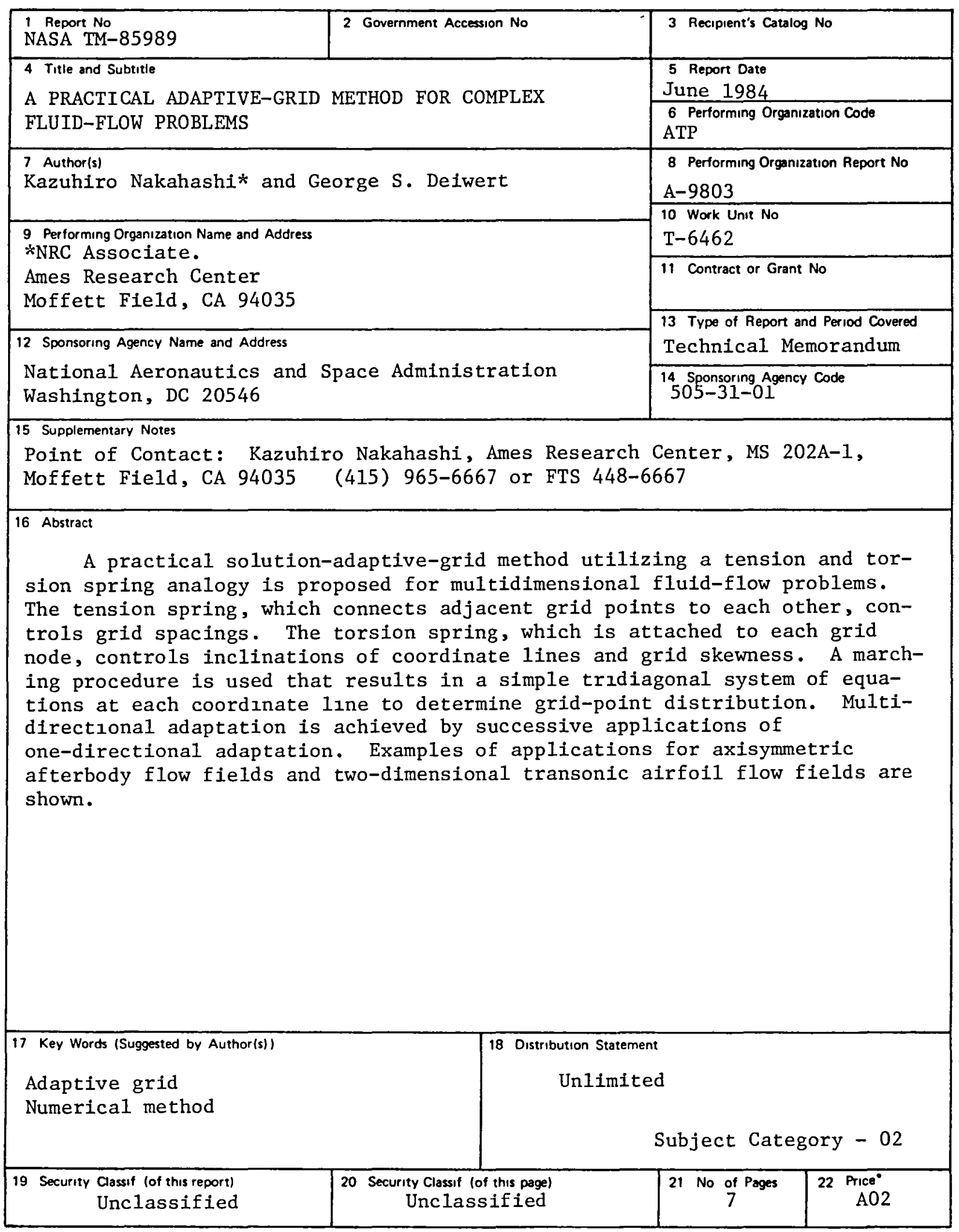

-For sale by the National Technical Information Service, Springfield, Virginia 22161 
End of Document 